\title{
Conceptualizing Vulnerability among Tanzanian School Children
}

\author{
S. Chrispina Lekule, L. Clinton Beckford \\ University of Windsor, Canada
}

\begin{abstract}
Over the past two decades Tanzania has experienced an increase in the number of vulnerable children and it has become a major issue of concern among educators, researchers, policymakers and communities which are highly impacted by this phenomenon. Due to the complexity of vulnerability among school children in Tanzania, this paper focuses on vulnerable children defined as being at high risk of either dropping out of school or facing academic failure due to vulnerable conditions which include, poverty, orphanhood, streetism, child abuse, and hostile family conditions. Hence, drawing from a Tanzanian perspective, this paper examines the conceptualization of vulnerable children and the factors that render them vulnerable. It also evaluates how Tanzanian schools could be of support to this population of children so that they could become successful in their education as a means to end vulnerability. The paper establishes that while the Government of Tanzania has done a lot in ensuring that every Tanzanian child has access to quality education, there are questions about the extent to which vulnerable children are catered to in the public education system. The paper also notes the lack of literature on what Tanzanian schools practically do in support of this growing population of children. To fill this vacuum, the paper analyzes several studies conducted in other countries with similar challenges and offer suggestions for what could be done by Tanzanian educators to improve the learning process and academic achievement for their vulnerable student population.
\end{abstract}

\section{Introduction}

The United Republic of Tanzania (URT) is one of 155 countries which have adopted the World Declaration for Education, which seeks to provide education for all children by the year 2015 [1]. Progress towards this achievement has been impressive at the primary level with net enrollment rising from 59\% in 2000 to $97 \%$ in 2008 [2]. However, only $34.9 \%$ out of $97 \%$ of the children enrolled in primary school transitioned to the secondary level [2]. These findings provide clear evidence of the inconsistencies that exist between primary and upper levels of education. It indicates that even if children manage to attend primary school, many do not succeed to the extent that they are able to move on to secondary school. Transition to secondary school in Tanzania is not automatic but is depend upon students' performance in a qualifying national examination. Failure to acquire education beyond the primary level jeopardizes students' life chances. Unfortunately, it is the already vulnerable children in marginalized communities that are most affected.

Our observation indicates that school dropout rates among vulnerable children remain high and the Tanzanian educational system is as yet unable to meet their academic needs. It is therefore urgent that Tanzanian educational leaders and policy makers move beyond the issue of access as the major criterion for educational reform [3]. The time has come for educational leaders, policy makers and practitioners to evaluate their own practices in order to bring about the needed changes to emancipate vulnerable children.

It is understandable that the increasing number of vulnerable children in Tanzanian is a potential threat to the nation's economy and to social well-being. In order to reduce this threat and maintain social stability, strategic intervention is urgently needed to lessen the impact of factors that increase the number of vulnerable children and also to help those already in the cycle of vulnerability to escape it. Mediation strategies require a well-planned and articulated process, leading to a clear understanding of the problem and how it can be addressed.

This paper discusses the issue of vulnerable children in Tanzania. The paper examines conceptualizations of vulnerability among school children in Tanzania and the factors which cause or render children vulnerable. It also explores the impact of vulnerability on student learning and the role of schools in mitigating and enhancing learning outcomes among vulnerable students.

\section{Conceptualizing vulnerability among children}

The concept of vulnerable children is "riddled with complexities" [4, p.79]. Defining vulnerability among children is epistemologically problematic because it is a complex issue that depends on a specific milieu [5]. If the term "vulnerable children" is thus dependent on a particular setting, it must automatically denote different things to different people. This is likely the reason why researchers have asked that local communities take responsibility for identifying factors in their context that cause vulnerability and use their own judgment for setting priorities for local action. It is from this background that for the purpose of this paper a definition that meets Tanzanian local standards for identifying 
vulnerable children is adopted. However, it must be noted that the definition included here does not consider children with developmental delays or physical disabilities. Instead it focuses on children who are at high risk of either dropping out of school or facing academic failure due to conditions that render them vulnerable such as, poverty, orphanhood including due to HIV/AIDS, streetism, child abuse, child labor, and hostile family conditions among others.

According to a situational analysis study conducted in 2002 which documented the experiences of orphaned and vulnerable children in Mwanza, Tanzania, vulnerable children are "those who are below the age of 18 and are living in very hard circumstances of life and are lacking many basic needs" (translation from Kiswahili, "Watoto wanao ishi katika mazingira magumu" [6, p.19]. Expanding on this definition, the study described the life situations of many Tanzanian children regarded as vulnerable. Among them were:

- Those who have been abandoned by one or both parents, including babies/young children who are left in public places (social orphans).

- Children living in the streets, town centres, bus stands or in fishing camps.

- Children who are working, especially undertaking hard labour.

- Children living in acute poverty and in broken families.

- Children living with irresponsible or uncaring parents or guardians.

In another study conducted in Tanzania to examine the link between poverty, HIV/AIDS, and education, the researcher defined the term vulnerable children as any person below 18 years who fit into one of the following categories:

- Children who are orphaned and without adult support

- The homeless and those living with families without minimum basic necessities

- Those living with critically ill parents and/or siblings.

[5].

Yet another definition of vulnerable children that may be applied can be found in a report by the United States Agency for International Development USAID (2008), in which the following categories are used to identify vulnerable children, mostly in developing countries:

1. Children whose safety, well-being or development is at significant risk.

2. Children orphaned due to AIDS or those infected with HIV or children caring for terminally sick parents or siblings.

In addition to these views, Lugalla \& Mwambo 1999 and Whitehouse, 2002 describe vulnerable children in terms of having little or very restricted access to basic needs, including education, family care and support, sufficient food and basic nutrition, protection from maltreatment, and neglect, as well those who do not have access to appropriate health care, and good hygiene. Based on these characteristics of vulnerability, it is crucial that proper care be given appropriately and in a timely manner. If this does not happen the result is that many vulnerable children will experience psychological, social, physical, and mental challenges which can in turn jeopardize their learning abilities and potential success in life. In this context we argue that educators need to be more aware of their vulnerable children and develop skills and strategies for meeting the learning needs of these children.

\section{Significance of the study}

The phenomenon of vulnerable children has received extensive attention in both academic and nonacademic circles but there has been little work on their educational experiences [4]. This creates a knowledge gap, which must be addressed as it has implications for development and human rights considerations.

The World Conference on Education for All in 1990 made clear that despite best efforts to meet basic learning needs, many countries continue to have notable and persistent challenges in ensuring the right to quality education for all. It was noted that millions of children (more than two third of them girls) did not have access to primary education (Ministry of Education and Culture, 2003) and those who did often did not achieve the level of education required to improve their lives and contribute to economic development. More than two decades later, many Tanzanian children especially the most vulnerable still do not have access to quality education [3]. This occurs despite Tanzania's recognition of the problem and efforts to achieve the Development Vision 2025, which highlights the issue of education for all as a fundamental strategy for development and a means to end the cycle of poverty and vulnerability [7]. Marginalized communities where vulnerable children usually come from, view school systems as first places were children could find support. Schools have a significant role to play in support of vulnerable children. Efforts are being made in Tanzania to ensure vulnerable children have access to school and community support. However, vulnerable children continue to lead in school dropout and poor academic achievement and there are gaps in knowledge about what we view as significant in meeting the academic needs of vulnerable children including: understanding of school experiences of vulnerable children; knowledge about how schools can support vulnerable children; challenges which teachers may experience as they deal with the increase of vulnerable children; 
and possible intervention strategies to meet the learning needs of vulnerable children. Given the current situation of vulnerable children in Tanzania, if proper intervention strategic steps are not taken this problem may have long term negative economic and social impacts on their immediate communities and the country at large. Hence, there is an urgent need to enhance the understanding of pedagogical and intervention strategies that will create better learning experiences for vulnerable children. This paper can inform policy about enhancement of student success among vulnerable children in Tanzania.

\section{Factors contributing to child vulnerability in Tanzania}

There is a growing number of vulnerable children in Tanzania. Many of these children are deprived of parental and/or adult care, love and supervision. Children who find themselves in vulnerable conditions are deprived not only of material needs but also of their very right to be children [6]. For many vulnerable children especially those who have been forced by their situation to abandon their homes to live on streets their childhood innocence may be shattered due to struggles for survival. In Tanzania, there are many factors that contribute to the sufferings of vulnerable children and they are not limited to unmet basic needs because some of their problems can also be associated to internalized problems of their past life which may have affected their psychological and social well-being.

Poverty and HIV/AIDS can be singled out as major factors that render millions of children in Tanzania vulnerable. Studies conducted in various parts of Tanzania have identified both material and non-material poverty as the root cause of child vulnerability. For example, in the Northern regions of Tanzania, including: Kilimanjaro and Arusha, Mwanza, and Dar es salaam, research have shown that many vulnerable children suffer tremendously due to lack of reliability of basic needs like access to food, shelter, health care, security, education among others $[8,6 ; 9]$. In one of these studies the authors clearly outlined the findings indicating that $100 \%$ of vulnerable children who were found homeless or living in desperate conditions in the city of Dar Es Salaam had abandoned their homes due to the general situation of poverty [8]. The same research indicated that $90 \%$ had left their homes due to lack of food, whereas $69 \%$ had abandoned their homes due to parent/matrimonial problems or death. Sixty two percent of the children in the study had left home due to domestic violence and abuse.

Other studies have identified orphanhood as a major factor in child vulnerability. It is important to note here that while the term, orphaned children is usually used to refer to those below the age of 15 or 18 in some cases, whose biological parent/s have died children who have been abandoned by biological parent/s are also considered orphaned [6]. This is a recognition of the fact that abandoned children are often no different from children who have lost their biological parents.

Studies conducted in Tanzania have also shown that orphaned children who lack parental or adult support are exposed to more risky conditions in which they may be deprived of their social and economic rights including the right to quality education [6]. Likewise, as noted by some scholars, orphaned children may be particularly vulnerable, because they do not have the emotional and physical maturity to bear with the nature of the suffering they face in their tender age and so they fail to address adequately the psychological trauma associated with their vulnerable conditions such as a loss of a parent [10]. In same vein UNICEF (2005) found out that orphaned children are frequently deprived of quality education, which would has been instrumental in protecting them from other vulnerabilities such as the possibility of contracting HIV/AIDS. In addition, orphaned children when in school are less likely to be at their correct grade compared to those who are not orphaned [6].

Orphaned children have been noted as having multiple disadvantages, which lead them to experience more serious problems, which put their lives in jeopardy and increase their vulnerability [11]. For example in a study comparing the educational status of orphaned and non-orphaned children in Tanzania and Burkina Faso, there was evidence of delayed enrolment of orphaned children and many did not start school at all. The study noted that in Tanzanian, $27.3 \%$ of sample of 4931 children started school late with an even greater number in Burkina Faso- $64.8 \%$ of 4835 . However, the study found that educational status of orphaned children was "relatively equivalent to non-orphaned" [11, p.726]. The researchers associated this condition to strategic efforts that were being made by both the community and families in support of these children. For example, the orphaned children in Tanzania were being supported through a multi sectorial program which works through a "combination of community support whose aim is to improve the individual living conditions or orphaned and vulnerable children in order to simultaneously improve the community environment where they live" [11, p.727]

Several studies have also associated causalities of domestic violence, sexual abuse, and gender discrimination, abuse of drugs and alcohol, as well as exploitation as some of the major factors that contribute to the pervasive and complex nature of the phenomenon of vulnerable children in Tanzania [6]. The issue of gender also comes to play as one of the factors that contribute to child vulnerability. Evidence of this claim has been confirmed in a recent study, which was conducted in Iringa, Tanzania with the 
purpose of documenting child domestic labor. The researchers concluded that child domestic labor was feminized. This means that the majority of children involved in child labor are girls. According to their findings, $73 \%$ of the children who participated in this research had entered into child labor at the age of 15 [12]. The study further noted that the major reasons for child vulnerability was mainly poverty, family responsibilities, parental pressure, lack of education, and family problems which included divorce and abuse. Children's struggle for survival and orphanhood were also identified as factors that contribute to child vulnerability in this study.

Klocker summed up the research findings noting that due to trauma, stigma, inadequate care, child labor, child abuse, which many children experience may eventually lead to psychological distress [12]. The findings of this study suggest that one type of vulnerability leads to another and factors of vulnerability are inter-connected rather than isolated. For example, Whitehouse noted that once a child lacks parental support, he or she may be forced to enter into a child labor situation where they often experience abuse and exploitation. Some children are forced by their situation to move from their immediate families and live with their extended family members [6]. Although this might seem to be a better option than becoming homeless, there is evidence that fear, anxiety, and poor treatment often lead such children to the streets.

Another major factor that has been identified as cause of child vulnerability is streetism.

The term streetism is a multifaceted concept, which encompasses conditions of children who are forced to abandon their homes to live on the streets usually of big cities and towns. According to Ajiboye, and Oladiti (2008) streetism is a situation in which children facing abandonment, exploitation and abuse are forced to live and sleep on the streets, which they view as "a means of survival as well as a place of abode" (p.126). In Tanzania children who are less than 18 years and are living as either full time or part time in the streets are a regarded as most vulnerable in the society $[13 ; 19]$. They also suffer from continuous harassment from civilians and law enforcement people [10]. It has argued been that it is difficult for a child who has been on the streets to regain normal life [9]. According to Woods, the life of children in the streets of Tanzania involves "rooting through rubbish bins for food, being shunned by locals and searching for drugs like cocaine, marijuana and glue which they use to ward off cold and hunger" [9, p. 1]. It can therefore be concluded that the lives of children who live on the streets is characterized by desperation for these basic needs and survival strategies. The tough conditions, which they experience often, force them to utilize different strategies which are often dehumanizing [8]. The nature of their livelihood strategies has led many local people to categorize them as 'Street Children.'

In our recent preliminary research orphaned and vulnerable children in Singida, central Tanzania, we found that vulnerable children are perceived in negative ways. They are often seen as hooligans, vagabonds and/or bad children who are prone to commit crimes and therefore deserve ill-treatment. Although there are many Tanzanians who empathize with them, from our observations in Singida these children are seen as nuisances. It has been reported that street children in Arusha are often rounded up and detained by law enforcement when high ranking people are visiting [9]. A good example of this took place in 2004 when the German President was visiting [9].

Similar trends of maltreatment and negative attitudes towards children who live on the streets have been noted by previous studies [8; 19]. Although this problem is huge and it seems to be affecting the whole structure and social life of the big cities, research has shown that some communities do not see this as their problem [6]. In some cities like Dar es Salaam, the children on the streets are viewed as a menace to the city [8]. Others regard them as "children with deviant behavior, as troublemakers or thieves [13, p. 23]. For this reason, these children face a lot of harassment from both community members and the police. As noted by Whitehouse children under this condition are still missing "almost every right of childhood from basic like food and medicine to assess to education and protection form exploitation and abuse" (p. 20).

There is evidence that children who spend their lives on the streets are well organized and are aware that people view and treat them as bad children [8]. Many of them are also aware that education could be the means for them to overcome the conditions that made them vulnerable and forced them into a more desperate life in the cities. For example, in one of the studies conducted in Dar es Salaam, one participating living one the streets told the researchers that,

Look here! My parents are very poor and that is why they did not send me to school. I am sure that if I was educated and had certain skills, I would not be living in the Way. I am also sure that if I get some training now, I will be able to manage my own life more easily [8, p. 341].

In a recent survey study which was conducted in Kilimanjaro and Arusha regions of Tanzania with the purpose of examining how child abuse or support factors may influence children migration into the street, McAlpine, et al. (2010) found out that children who were abused by their parents or care givers and did not feel safe and supported at home had the tendency of migrating into the cities where they expect to make better lives. According to a local NGO, children who find themselves struggling in 
their home villages due to poverty, abuse or other reasons "believe that better life is available in urban areas and so flock to town under the impression that, "streets are paved with gold" [3, p. 7]. This finding would suggest that children living on the streets are pushed away from their homes due to both material and non-material poverty. Other reasons included hunger, family and neighborhood violence, family dissolution, and in the breakdown of traditional supportive community structures [13].

Structural causes also lead to child immigration to the streets including, rapid population increase, an unresponsive employment market, an under-resourced educational system, increased pressure on peasants and an increasingly uneconomic smallholding in the rural sector. Given the kinds of experiences and the circumstances, which render children vulnerable it is worthwhile to consider how vulnerability impacts children's school experiences.

\section{Addressing the academic needs of vulnerable children: Educators' roles and strategies}

In a study of street children in the city of Dar-essalaam it was noted that vulnerability affects children's learning [8]. Williams (2010) concur with this view indicating that vulnerable children should be given maximum support to overcome anxiety fear and other consequences of vulnerability, which have negative impact on their learning process. Similar studies, which have been conducted in some large cities of Tanzania such as Arusha, Moshi, Tanga, Mbeya and Mwanza, have also found that vulnerable children's experiences negatively influence their learning and ability to meet their potential.

Thus, addressing the academic needs of vulnerable children is an absolute necessity. Generally, Tanzania has in recent years, taken significant steps to address the educational needs of vulnerable children [10]. In 2002, for example, the government abolished primary school fees to guarantee access to basic education. Learning environments and teacher training were also improved to ensure quality in teaching. In addition to these efforts and in recognition of the critical needs of vulnerable children, the Tanzanian government has enacted a number of measures specifically aimed at benefiting vulnerable children. For example, in 1995, the government initiated the Most Vulnerable Child (MVC) program with the intention of involving communities in contributing to the improvement of care and support to vulnerable children living in their midst. In 1999, the government collaborated with UNICEF to enact the Complementary Basic Education in Tanzania (COBET) program, intended to provide educational opportunities for school children aged 7-18, with special focus on girls and vulnerable children [7]. According to a report issued by
UNICEF, these programs have contributed to shaping national policy and practice, and have had a positive impact on vulnerable children [1]. However, much still needs to be done in the schools for actual realization of these policies.

At different occasions including the World Educational Forums in 1990 and 2000 which took place at Jomtien, Thailand, and in Dakar Senegal respectively, as well as the World Economic Forum in Davos, Switzerland in 2005, educators, business and political leader all recognize education as the key to uplifting humanity and for alleviating poverty and vulnerability. For this reasons, it is important for educators to make sure they are capable of identifying issues facing vulnerable children in their schools and find specific strategies for supporting them [10]. Vulnerable children go through a host of traumatic experiences in their lives. Experiences like physical or sexual abuse, abandonment, exposure to hostile conditions and domestic violence, loss of a parent or care giver and the like, will hardly disappear from the child's memory. It is also true that those children who are living with such painful memories could have difficulty learning. Studies have shown that there is likelihood for vulnerable children to suffer posttraumatic stress (hyperarousal) which can lead to serious lack concentration while in class and at times they experience feelings of anxiety, anger, hate and fear [14]. Cohen and Mannarino further explained that vulnerable children who suffer from emotional and psychological imbalance due to their past experiences will prospectively develop a tendency of withdrawal from social activities especially if they view them as a reminder of their past negative experiences. In a recent quantitative study of 306 children, which examined emotional status of vulnerable children, it was found that $85 \%$ of them had experienced emotional distress and had often acted with anger [15]. In this same study, $71 \%$ reported that they had often or sometimes experienced scary dreams. Likewise, $71 \%$ among the same group admitted that they were troubled by worries. The study also found that another $71 \%$ had experienced feelings of unhappiness or sadness while $67 \%$ agreed that they had often preferred to be alone [15, p.196]. These findings confirm that traumatic experiences will always remain with the children and will at one time appear in a variety of ways which are hard to determine and will affect children' learning.

Considering the nature of the above-mentioned status of vulnerable children it is apparent that educators face serious challenges in meeting their academic needs effectively. Some researchers have written to remind schools of their roles for children's academic and psychosocial development [10]. These scholars emphasize the significance of educator's awareness of the presence of vulnerable children in their schools and also their ability to identify different strategies which can be utilized individually or 
communally at school level as a means to support and ensure effective learning for all [10].

A couple of studies have examined the type of strategies, which some schools have successfully utilized in supporting vulnerable children. For example, in New Zealand, Mutch, Rarere, \& Stratford conducted case studies involving eleven primary schools which were considered exemplary in the efforts for supporting their children. School principals, teaching staff, support staff school social workers and parents were recruited to participate in in-depth interviews, which focused on the type of strategies, which they might have employed in supporting in vulnerable children their schools. For the purpose of reliability these researchers also conducted a critical analysis of "written documents, school charters, school and classroom planning, assessment data, and school policies and procedures" [16]. After analyzing data by means of comparative analysis these scholars concluded that participating schools had well-developed strategies for supporting vulnerable children contributed to the high-quality teaching and learning which was evident in these schools. The study concluded with a suggestion that all schools must consider how to provide effective support to their vulnerable children. Among the strategies identified was the presence of a positive school culture which participants defined simply as the "way things were being done at school" [16, p. 323]. The study found that some schools developed positive school culture as a means of making vulnerable children comfortable and confident in their learning process. Accordingly, the participating schools had norms that prioritized a safe caring and inclusive school culture. They also had beliefs and values that were based on building friendly and welcoming learning environments [13]. As a matter of fact, McGrath and Noble also identified the aspect of maintaining a safe and inclusive culture of caring for all students as a starting point that will prevent vulnerable children in the school from withdrawing from social activities. They suggested that maintaining positive relationship among school stakeholders can contribute to strengthening support efforts for vulnerable children that will lead to a production of desirable learning outcomes.

Other aspects related to establishing a positive, inclusive school culture as a strategy for supporting vulnerable children were also identified by researchers [10]. They identified factors like: the presence of a policy for identifying vulnerable children and follow-up; home school communication; providing one on one support; encouraging supportive peer network; equipping teachers with appropriate strategies and knowledge needed to effectively support vulnerable children [10]. Mutch et al have also added to this list through their findings where schools had "thorough processes identifying and removing the barriers to achievement faced by students and also placing priority on finding and developing the strengths of all students" (p. 234). Other aspects related to school culture that can be used as a strategy for supporting vulnerable children were also highlighted. One of these was finding and developing the strengths of all students. Under this strategy, schools developed programs and cultural practices that provided students with opportunity to utilize particular strengths as a means enhancing their feelings of inclusion and value [16]. The tradition of being and maintaining responsiveness to social and educational needs of vulnerable children was another strategy found common among schools that participated in this study. It was noted that educators were taking "genuine responsibilities for supporting students and improving their learning outcomes" [16, p. 235]. In relation to this strategy it was noted that teachers did not accept low levels of achievement and so they worked in the context of the school's welldeveloped systems for care and special education, to manage social and academic issues of their students [16].

More practical strategies such as negotiating with and supporting vulnerable children in their homework and encouraging peer support were also identified in many successful schools [10]. Educators as well as students at different capacities worked together in reinforcing positive school culture of supporting and budding students as a means of helping those who needed support.

School leadership is another aspect under which major strategies for effectively supporting vulnerable children evolve. Mutch et al noted in their study that starting from school principals down to teachers, staff members and student leadership, they all work together to determine how to best support vulnerable children. According to Mutch et al., school leadership that used a strategy of leading as a team played a great role in establishing and ensuring support for vulnerable children. More specifically, school principals played specific roles in maintaining a positive school culture such as: placing priority on meeting the needs of all students and ensuring that students received support and resources; linking families and communities; conducting interviews with families and remaining connected to any ongoing inter-agency support; maintaining team work and strong collaboration in supporting students and ensuring appropriate support was given to the children that needed it.

In a developing country like Tanzania where education is viewed as the most reliable strategy for eliminating poverty and vulnerability, educators must embrace critical pedagogy promote a holistic approach to education. By this we suggest that, educators will have to play a significant role in providing an education that supports and leads all learners to emancipation and thereby prepare them for 
active participation in their society. Knowlton argues that teachers must ensure higher attainment of appropriate education of vulnerable children. There are many ways in which teachers and school leaders can provide invaluable support that will make a difference in the learning process of vulnerable children. As a means to encourage and nurture the learning experience of vulnerable children, Knowlton suggests that teachers consider approaches, such as; (a) being sensitive to the feelings of vulnerable children and creating a climate of acceptance and understanding; (b) offering other students as a buddies to the vulnerable children who are new in the school or those who are going through difficult moments; (c) allocating mentors for the vulnerable; (d) providing educational accommodation to the child with an expectation of improving; (e) conduct one on-one tutoring as a vital means for building self-esteem and encouraging learning.

School principals and other administrators play a central role in leading the whole school system towards making a difference in the lives of vulnerable children [17]. They are expected to mobilize and to be role models as a means of encouraging others to journey together in pursuing school goals including that of supporting vulnerable children. More appropriately, school leaders should be concerned with influencing and setting a direction in support for vulnerable children. According to Knowlton, the following are fundamental roles of school leaders. First, school leaders should ensure that appropriate educational and school support services such as school counseling, tutoring and after school programs are in place. Second, they should train teachers and support personnel on how to help vulnerable children. Third, make sure that teachers and support staff know and practice policies and laws that protect the rights of vulnerable children in the process of providing quality and equitable education. Fourth, school leaders should endeavor to create a working relationship with nearby civil and religious organizations that offer support to the needy and also collaborate with community to provide needed help. Fifth, it is the leaders' responsibility to appoint liaisons for the vulnerable children. The key role for such liaisons is to link local education agencies to community resources. The importance of educators support for vulnerable children cannot be overstated. If vulnerable children are to overcome the difficult circumstance in which they find themselves, educators must establish concrete strategies through which they can provide maximum support to each vulnerable child in accordance to each one's particular needs.

Another strategy linked to school leadership in some studies is criteria used by schools when appointing new teachers and staff. For example, Mutch et al. found that "school principals were very careful in matching the values of the school with the appointment of staff' (p. 237). In this study, there was evidence that strategies for supporting vulnerable children were long planned beginning with criteria for appointing employees in the school who were expected to implement school strategies for supporting vulnerable children. In one of the school, a school principal told these researchers how, they would not consider recruiting a new staff member who did not portray leadership qualities, related to commitment to meeting the individual needs of students and valuing of their diverse backgrounds. The teacher added that those applying to work in their school should portray the ability to build excellent relationships with students and their families, and also demonstrate that they were capable of working collaboratively with other staff members in supporting vulnerable children in their school [16].

Student leadership has also being found as another strategic means of meeting the needs of vulnerable children. The roles played by school leaders involved participating in the school council and also supporting and buddying with students that needed help [16]. This strategy of utilizing student leadership has been identified by other studies as a powerful means of improving student well-being and academic outcomes [18]. They argue that, "the more pupils are actively engaged and achieving learning the greater their sense of well-being and vice versa" [18, p. 79].

\section{School as a caring community}

If I have seen further, it is because I have stood on the shoulders of a giant (Isaac Newton)

Tanzania is known to have a strong commitment to ensuring the realization of education for all and yet based on the nature of challenges facing the educational system the progress of achieving this goal is much slower than anticipated [19]. In some regions of Tanzania school enrollments are declining a fact that is difficult to understand in this era where education is claimed to be right of every child. Okkolin et al. argue that the reason for such decline is "abject poverty among parents who fail to meet the educational costs, long walking distances to school, inadequate curricular, parents who attach little value to education, and lack of space especially in urban schools such as Dar es salaam"(p. 72). Based on our experience working with vulnerable children in rural Tanzania, vulnerable children who come from poor rural home without basic necessities such water, electricity, sufficient food, are always happy to be in school especially when they received care and feel safe there. Many of them see school as a home away from home. They come to school with high expectations of finding what they do not find at home. Vulnerable children often display fear and anxiety about whether the school would be a safe place where they can find supportive relationship from peers and 
their educators. This is fundamental in shaping their motivation to learn. For this reason, educators must be keen enough in anticipating the individual learning needs of all children. They need to know that children come from different backgrounds and that this requires accommodation. With this view, we suggest that educators create a learning environment that protects the child and supports their developing needs thereby fostering characteristics that will increase resilience in all children. Apart from the role of providing teaching and learning, schools should also be capable of offering services to the community especially to children with special needs. Schools are the most strong and reliable social systems in the communities for this reason they have important roles and responsibilities towards vulnerable children. They should make sure that they offer epistemological education to students. What this entails is that schools have the responsibility of providing access to learning which is not limited to theoretical perspectives but rather one that focuses on providing an education which can inspire learners to re-imagine their future and how it can be attained. This role should be considered as one of the most significant, because it is through such roles that the needs of vulnerable children can be met. In the rural communities of Tanzania, schools are the only public institutions that community members can rely on. For this reason schools ought to be organized and managed in a very welcoming manner so that communities can feel comfortable in going to them as their resource centers where their vulnerable children can receive adequate and reliable services and support which they urgently need. In other words, schools should be a pathway through which vulnerable children's lives are improved and a source of assurance of a better future.

Research has shown that an excellent approach in educating vulnerable children is for educators to be sources of hope and stability in the lives of vulnerable children [12]. Unfortunately, there is uncertainty regarding the manner and the extent to which schools, especially those in the developing countries like Tanzania, are signs of hope and stability to vulnerable children. For example, in a study conducted in South Africa, teachers and school leaders expressed doubts about their ability to meet the challenge of supporting vulnerable children and that nothing was done to support such children [18]. Such findings and the lack of confidence and certainty is an indication that today's vulnerable children are still an invisible population in a school setting.

Another study which was conducted in three African countries, namely, Malawi, Zambia and Zimbabwe, found that there were incidences related to vulnerable children causing significant interruptions to the school routine [20]. For example, students missing school, acting up in the class, getting physically weak or emotionally disturbed, to name a few. Despite all the disruptions experienced by schools in relation to issues surrounding vulnerable children, the study found that the schools involved did not consider the situation of vulnerable children an issue to be discussed at committee meetings, Parent Teacher Association (PTA) meetings or internal school planning meetings [20]. Vulnerable children have particular needs and require educational intervention that will enable them to overcome emotional stress, anxiety, fear, and hopelessness [20]. Therefore, in order to provide vulnerable children with quality education, school leaders should create programs that promote regular school attendance. In a study conducted by the United Nations Children Education Fund assessing and documenting profiles and identifying the educational needs of children in two districts in Tanzania, among the factors noted as hindrances for vulnerable children to enrol or to remain enrolled in school leave alone succeeding were:

1. Many out-of-school children were unable to commit to attending formal school, which meets for six hours a day, five days a week.

2. Many were unable to afford school uniforms or materials.

3. Existing school environments were unlikely to attract out-of-school children into - or back into the school system. The general state of buildings was often found to be poor.

4. Syllabi, textbooks and pedagogy designed for age-appropriate teaching to children in different grades (e.g., Standard 1 materials designed for 7year-olds) were often unsuitable for teaching older children taking earlier classes (e.g., 16year-olds in Standard 2).

5. Many students lived far away from available schools. [1]

\section{Conclusion}

Despite well documented and commendable efforts of the Government of the United Republic of Tanzania, to provide quality education for all, there is still a big gap between policy and actualization. Especially in the case of vulnerable children, the public education system is struggling to cater to the needs of its students. There is evidence from the literature that vulnerability has deleterious impacts on student learning and that schools are generally illequipped to meet the needs of vulnerable children. We argue that this issue is so important that it is imperative for policy-makers to bolster guidelines and protections for vulnerable children generally and more specifically in the school system. More importantly it is important for educational practitioners to make sure that the gap between policy and implementation is closed. That being said there is very little research on the relationship between vulnerability and student learning in Tanzania. More research in this area is a fundamental need at this time. 


\section{References}

[1] UNICEF, Contextualizing child poverty in Tanzania: Legal framework, social services and the state of HIV/AIDS and malaria, 2007, pp.132,http://www.gpia.info/files/practicum/28/2007\%20Fall\%2 0\%20UNICEF\%20\%20Global\%20Child\%20Poverty\%20Di sparity\%20and\%20Tanzania. pdf (November $3^{\text {rd }}, 2011$ ).

[2] USAID Education programming for orphans and vulnerable children affected by or vulnerable to HIV: Moving beyond school fees and uniforms final,2008,pp.166 http://pdf.usaid.gov/pdf_docs/PNADP105.pdf (November $3^{\text {rd }}, 2011$ )

[3] H. Johnson, T. Kisslinger, S. Oneko, \& A. Heuser, Response to child Vulnerability: Why children migrate to the streets in Tanzania. 2005, pp.1-27. http://www.mkombozi.org/publications/research (July, 20th 2012).

[4] L. E. Eloff, \& J. Viljoen, Re-conceptualizing vulnerable children by acknowledging their assets. African Journal of AIDS Research (AJAR), 6(1), 2007, 79-86.

[5] R. Evans, Poverty, HIV, and barriers to education: Street children's experiences in Gender \& Development, 10 (3), 2002, pp. 51-62

[6] A. Whitehouse, A situation analysis of orphans and other vulnerable children in Mwanza Region, Tanzania. 2002, pp. 1-85.

[7] Ministry of Education and Culture, Girls' education: Tanzania at a glance, 2003, pp.1-2, http://moe.go.tz/pdf/Plans\%20Programmes\%20and\%20proj ects.pdf (January $\left.15^{\text {th }}, 2012\right)$

[8] J. P. Lugalla, \& J. Mbwambo, Street Children and Street Life in Urban Tanzania: The culture of surviving and its implications for children's health. International Journal of Urban \& Regional Research, 23(2), 1999, pp. 329 -344

[9] J. Wood, Change a life. Lawyer, 19(49), 2005, pp. 1819.

[10] A. Reupert, \& Mayberyb, Strategies and issues in supporting children whose parents have a mental illness within the school system. School Psychology International, 28(2), 2007, pp. 195-205.

[11] M. L. Kurzinger, J. J. Pagnier, J. G. Kahn, R. R. Hampshire, T. T. Wakabi, \& T. V. Dye, Education status among orphans and non-orphans in communities affected by AIDS in Tanzania and Burkina Faso. AIDS Care, 20(6), 2008, pp.726-732.

[12] N. Klocker, Negotiating change: working with children and their employers to transform child domestic work in Iringa, Tanzania, Children's Geographies, 9(2), 2011, pp. 205-220.

[13] K. McAlpine, R. Henley, M. Mueller, \& S. Vetter, A survey of street children in northern Tanzania: How abuse or support factors may influence migration to the street. Community Mental Health Journal, 46(1), 2010, pp. 26-32.

[14] J. A Cohen, \& A. P. Mannarino, Supporting children with traumatic grief: What educators need to know, School Psychology International, 32(2), 2011, pp.117-131.

[15] S. M. Kirkpatrick, W. Rojjanasrirat, B.J. South, J. A. Sindt, \& L. A. Williams, Assessment of emotional status of Orphans and vulnerable children in Zambia. Journal of Nursing Scholarship, 44(2), 2012, pp.194-201.

[16] C. Mutch, V. Rarere, \& R. Stratford, 'When you looked at me, you didn't judge me': Supporting transient students and their families in New Zealand primary schools. Pastoral Care in Education, 29(4), 2011, pp. 231-245.

[17] A. S. Knowlton, Helping the homeless: What educators can do to ease the pain, Delta Kappa Gamma Bulletin, 73(1), 2006, pp. 17-19.

[18] McGrath, H., \& Noble, T. (2010). Supporting positive pupil relationships: Research to practice. Educational \& Child Psychology, 27(1), 79-90.

[19] M. Okkolin, E. Lehtomäki, \& E. Bhalalusesa, The successful education sector development in Tanzania comment on gender balance and inclusive education. Gender Education, 22(1), 2010, pp. 63-71.

[20] S.E. Williams, Exploring the viability of school-based support for vulnerable children: A case study of two township schools in Johannesburg. Consortium for Research on Educational Access, Transitions and Equity, 46, 2010, pp.1-48. 